\title{
Análisis cualitativo de un modelo de pesquerías de acceso abierto*
}

\author{
EDUARDO GONZALEZ O. y JAIME MENA L. \\ Instituto de Matemáticas \\ Universidad Católica de Valparaíso \\ Casilla 4059, Valparaíso, Chile
}

\begin{abstract}
RESUMEN. Un análisis cualitativo de un modelo bioeconómico de pesquería de una sóla especie es desarrollado usando una función de captura más realista que la usada en la hipótesis de Schaefer. Se discute la existencia de bifurcaciones en los puntos de equilibrio y su estabilidad. Se comenta el comportamiento de este modelo de pesquería de acceso abierto bajo el cambio de los parámetros. Una variedad de situaciones tanto biológicas como económicas son discutidas considerando el equilibrio bioeconómico y niveles de extinción, agotamiento del recurso, como también la autorregulación de la pesquería. Se presentan diversas curvas del espacio de fase del sistema usando el programa PHASER.
\end{abstract}

Palabras claves: capturabilidad, coeficiente de capturabilidad, esfuerzo de pesca, pesquería de acceso abierto, equilibrio bionómico.

\section{Qualitative analysis of an open-access fishery model}

\begin{abstract}
A qualitative analysis of bioeconomic model of a single species fishery is developed using a harvest function that is more realistic than the Schaefer hypothesis. The existence of a bifurcation of steady states and its stability are discussed. Several comments are made regarding the behavior of this model of open-access fishery under changes of some parameters. A variety of complexities, both biological and economic, are also discussed including the bioeconomic equilibriums and the level of extintion, collapse of the population as well as the self-control of the fishery. Diverse curves of the phase portrait are shown.
\end{abstract}

Key words: Catchability, catchability coefficient, fishing effort, open-access fishery, bionomic equilibrium.

\section{INTRODUCCION}

En este trabajo se presentan algunos aspectos de la estabilidad del equilibrio de un modelo de pesquería de una sola especie, en que el tamaño de la población y el esfuerzo de pesca varían continuamente con el tiempo. Se analiza la interpretación bioeconómica, que implica el empleo de una función de captura» que es más flexible y parece ser más realista» (Chaudhuri y Johnson, 1990), que la usada en la conocida y cuestionada hipótesis de Schaefer, sobre la «captura por unidad de esfuer- zo», y la que ha sido empleada en el manejo de diversas pesquerías comerciales.

Este tema resulta de singular importancia y de gran dificultad, puesto que se da origen a un modelo multiespecie. Al considerar el esfuerzo variable, cualquier pesquería de una sola especie pasa a conformar un modelo de interacción de especies, en que la especie explotada es considerada como la presa y el hombre como su depredador. La principal diferencia con otros sistemas depredador-presa, está en de la

\footnotetext{
Financiado por Proyecto DGI-UCV 124710/92 y Proyecto FONDECYT Nº 0028/92.
} 
la escala de tiempo de la evolución de la tecnología humana destinada a la pesca, comparada con la evolución del comportamiento de los depredadores naturales (Clark, 1985). Por otra parte, la voracidad del depredador, esto es, el esfuerzo de pesca empleado por el hombre, depende de otros factores, como por ejemplo, el precio del mercado y obviamente de las ganancias que reporta la actividad pesquera.

En el modelo se utiliza la ecuación logística para representar la dinámica de la población de peces, pero ésta puede ser cambiada por cualquiera otra función de crecimiento que tenga un comportamiento cualitativamente similar (funciones compensatorias), como son las utilizadas en los llamados «modelos globales de producción», obteniéndose con ellas resultados parecidos.

El sistema de ecuaciones diferenciales no lineales ha sido analizado principalmente en los puntos de equilibrios del primer cuadrante $\mathrm{r}=\left(\mathrm{R}^{+}\right)^{2}$, porque en esta región se tiene un claro sentido biológico. En el estudio se han utilizado diversas técnicas matemáticas, pero aquí sólo se presentan las conclusiones bioeconómicas más interesantes.

Las aplicaciones prácticas de este modelo se orientan a la regulación de la pesquería a través de agencias especializadas, implicando que tanto ellas como la industria misma, deben contar con el mayor caudal de información posible acerca de esta actividad económica.

\section{PROPOSICION DEL PROBLEMA}

Chaudhuri y Johnson (1990) acogiendo una sugerencia de Clark (1979) proponen una función de captura más flexible y que también resulta ser más realista que la hipótesis de Schaefer, la cual supone que la «captura por unidad de esfuerzo (CPUE), es proporcional a la biomasa», i.e.

$$
h(t)=Q \quad E \quad x=F x
$$

donde $\mathrm{x}$ indica la biomasa de peces (o densidad de población o fracción explotable de la población) y E es el esfuerzo nominal de pesca, es decir, la cantidad normalizada de embarcaciones y aparejos de pesca que están activas en la pesquería en un instante t dado, medido en SFU (unidades estandarizadas de pesca). Esta normalización es necesaria para tomar en cuen- ta las variaciones efectivas de los diferentes tamaños y tipos de embarcaciones, redes, etc. (Clark, 1985).

Por su parte $\mathrm{F}$ expresa el coeficiente instantáneo de mortalidad por pesca, y se tiene claramente que $\mathrm{F}=\mathrm{Q} \mathrm{E}$, por lo cual en este trabajo, no es necesario hacer una distinción entre $\mathrm{E}$ y $\mathrm{F}$, puesto que se ha realizado sólo un estudio cualitativo.

La función considerada en este caso para representar la razón de mortalidad por captura de la población de peces es:

$$
h(x, E)=\frac{q E x}{a E+1 x}
$$

El cuociente $(x, E)=\frac{q}{a E+1 x}$ indica la modificación al coeficiente de captura constante que aparece en la hipótesis de Schaefer. Expresa que el coeficiente de captura q es variable con el cambio de la biomasa y del esfuerzo y que varía en forma inversamente proporcional a la abundancia del recurso y al esfuerzo. Se ve claramente, que a mayor biomasa disminuye el coeficiente de captura q, lo que también sucede cuando aumenta el esfuerzo [situación que se cumple cuando las unidades de pesca o embarcaciones compiten entre sí (Yáñez y Maritano, 1983)].

De este modo se recoge la frecuente objeción a los modelos que consideran fijo este valor. Sin embargo, aquí la constante q tendrá el mismo significado anterior, ya que su variabilidad es considerada en la relación funcional indicada. Las constantes a y 1 tienen unidades de medidas tales que permiten sumar las variables involucradas.

Se debe hacer notar que para esta nueva función de captura se cumple que:

$$
\begin{aligned}
& \text { i) } \operatorname{Si} \mathrm{E} \rightarrow \infty \text { entonces } \mathrm{h}(\mathrm{t}) \rightarrow \frac{\mathrm{q}}{\mathrm{a}} \mathrm{x} \\
& \text { ii) Si } \mathrm{x} \rightarrow \infty \text { entonces } \mathrm{h}(\mathrm{t}) \rightarrow \frac{\mathrm{q}}{\mathrm{l}} \mathrm{x}
\end{aligned}
$$

es decir, la función de captura considerada recoge también la idea de saturación tanto con respecto al esfuerzo de pesca $\mathrm{E}$, como respecto a la biomasa $\mathrm{x}$ (Chaudhuri y Johnson, 1990).

En este modelo se supone que la biomasa de peces tiene un comportamiento descrito por la ecuación de crecimiento logística 


$$
x^{\prime}=\gamma\left(1-\frac{x}{K}\right) x
$$

Tal como lo hace Smith en Clark (1979), se asume que el nivel de esfuerzo E debe variar continuamente en forma proporcional a la renta neta percibida (beneficio económico neto) por la industria pesquera en ese instante, esto es,

$$
E^{\prime}=b(\pi h-c E)
$$

donde $\pi=\mathrm{p}$-T es el ingreso neto por cada unidad de peces desembarcada. Se obtiene, de este modo, el siguiente sistema de ecuaciones diferenciales no lineales:

$$
\begin{gathered}
x^{\prime}=\left[\gamma\left(1-\frac{x}{K}\right)-\frac{q E}{a E+1 x}\right] x \\
E^{\prime}=b\left[-c+\frac{(p-T) q x}{a E+1 x}\right] E
\end{gathered}
$$

Las constantes, todas positivas, tienen los significados bioeconómicos usuales.

$$
\begin{aligned}
& \gamma= \text { potencial biótico de la especie o tasa de cre- } \\
& \text { cimiento intrínseco. } \\
& \mathrm{q}= \text { coeficiente de capturabilidad. } \\
& \mathrm{K}= \text { capacidad de soporte del medio ambiente. } \\
& \mathrm{p}= \text { precio por unidad de biomasa de peces des- } \\
& \text { embarcada. } \\
& \mathrm{T}= \text { impuesto cobrado por una agencia regu- } \\
& \text { ladora por cada unidad de biomasa de peces } \\
& \text { desembarcada, } \\
& \mathrm{c}= \text { costo de pesca por unidad de esfuerzo. } \\
& \mathrm{b}= \text { parámetro de rigidez; mide la rapidez de la } \\
& \text { reacción del esfuerzo E, cuando cambia la } \\
& \text { renta percibida. } \\
& \mathrm{a}= \text { constante que es proporcional al cuociente } \\
& \begin{array}{l}
\text { entre el nivel de población y la captura, cuan- } \\
\text { do el nivel de esfuerzo es alto. }
\end{array} \\
& \mathrm{l}= \text { constante que es proporcional al cuociente } \\
& \text { entre el nivel de esfuerzo y la captura, cuan- } \\
& \text { do se tiene una gran biomasa. }
\end{aligned}
$$

Mediante la redefinición de las variables y la agrupación de los 9 parámetros, el sistema (2) se simplifica para su análisis, obteniéndose el sistema «adimensional» con sólo tres parámetros (González y Mena, 1991a) y equivalente matemáticamente al anterior, que se describe en las siguientes ecuaciones:

$$
\begin{array}{r}
\dot{x}=\left[A(1-x)-\frac{E}{E+x}\right] x \\
\dot{E}=B\left[-C+\frac{E}{E+x}\right] E
\end{array}
$$

con

$$
A=\frac{a \gamma}{q}, \pi=p-T, B=\frac{a b \pi}{l}, C=\frac{c l}{q \pi}
$$

$\mathrm{y}$ el punto sobre las variables $\mathrm{x}$ y $\mathrm{E}$ indica un reescalamiento en el tiempo dado por $\tau=\frac{\alpha \gamma}{q}$, por lo cual $x_{(n)}=\frac{x^{(v)}}{K} y E_{(n)}=\frac{a E^{(v)}}{1 K}$ donde (n) indica la nueva variable y $(v)$ indica la variable original. Claramente, los tres parámetros $\mathrm{A}, \mathrm{B}$ y $\mathrm{C}$ resultan ser también positivos.

El hecho que se pueda reducir la cantidad de parámetros a sólo tres, implica que para el estudio cualitativo no es necesario conocer cada uno de los nueve originales, sino que basta establecer o conocer las relaciones existentes entre ellos. Esta simplificación resulta de interés, puesto que se orienta la investigación a la búsqueda de relaciones entre los parámetros significativos de la pesquería, y además, la modelación indica cuáles de estos son los más relevantes y que deberían ser controlados o tomados en cuenta, cuando se está interesado en el manejo óptimo del recurso.

\section{RESULTADOS}

Los resultados obtenidos, cuya fundamentación matemática se da en el Anexo I, se expresan en términos de los parámetros originales en las siguientes proposiciones:

\section{Bioteorema 1}

a) Cuando q $\pi<$ c 1 y a b $\pi \leq 1$, la biomasa siempre alcanza su máximo valor $\mathrm{K}$, independiente del esfuerzo inicial E que se haga (Fig. la).

b) Si q $\pi<$ c 1 y a b $\pi<1$, existe un nivel de extinción $\mathrm{E}_{\mathrm{u}} \mathrm{y}$ se obtiene que para algunos niveles iniciales de esfuerzo, la biomasa es agotada y para otros la población alcanza su valor máximo K (Fig. lb). 

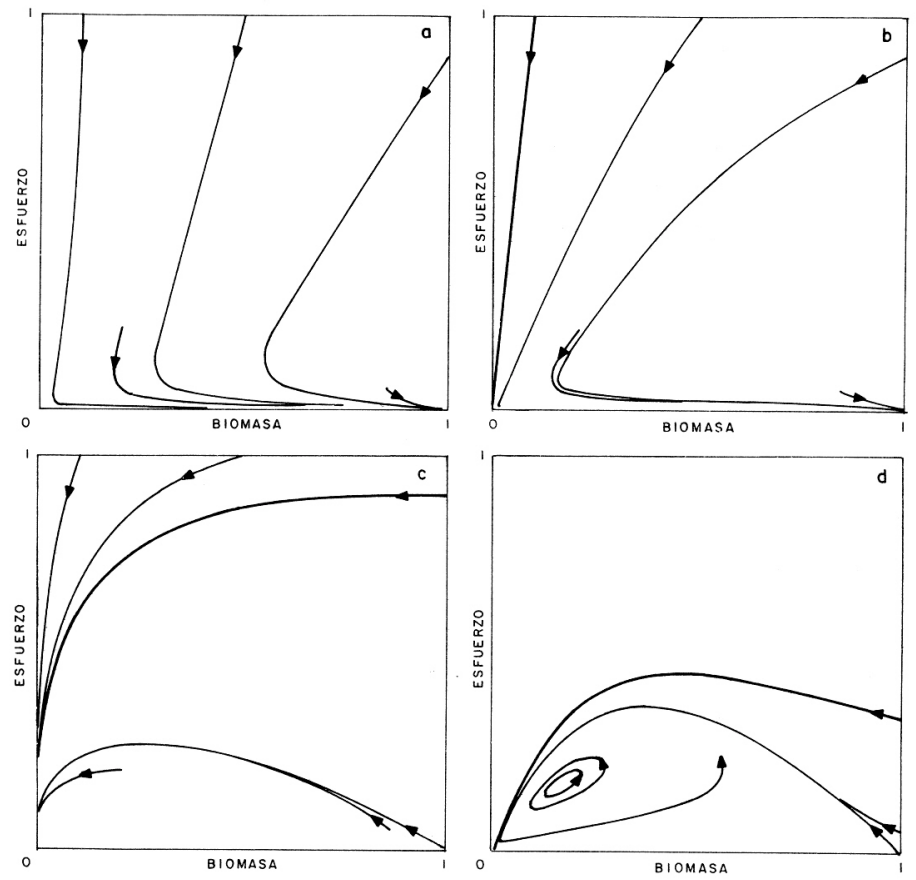

Figura 1. a) Recuperación de la biomasa $(a=0,4 ; b=1,2 ; c=1,2) ; b)$ curva de extinción y recuperación de la biomasa $(a=0,4 ; b=0,4 ; c=1,2) ; c)$ colapso de la pesquería $(a=0,4 ; b=0,4 ; c=0,5) ; y d)$ autorregulación oscilatoria y curva de extinción $(a=0,6 ; b=0,6 ; c=0,5)$.

c) Cuando a $\pi \gamma<\mathrm{q} \pi-\mathrm{cl}$, cualquiera sea el esfuerzo de pesca, la pesquería colapsa y el esfuerzo de pesca tiende a 0 (Fig. 1c).

Cuando el punto de equilibrio no trivial $\mathrm{P}_{\mathrm{e}}$ está en el primer cuadrante, coexiste con los puntos $(0,0)$ y $(K, 0)$ y se debe cumplir simultáneamente que:

$$
\mathrm{q} \pi<\mathrm{cl} \mathrm{y} \mathrm{a} \gamma \gamma<\mathrm{q} \pi-\mathrm{cl}
$$

Se obtiene que la traza de la matrizjacobiana en $\mathrm{P}_{\mathrm{e}}$ es:

$$
\begin{aligned}
& \operatorname{TrM}_{e}=1-C^{2}+B C^{2}-B C-A \\
& =\frac{1}{q^{2} \pi^{2}}\left[(q \pi-c l)(q \pi+c l-a b c \pi)-a \gamma q \pi^{2}\right]
\end{aligned}
$$

y se cumplen las afirmaciones siguientes:

\section{Bioteorema 2}

Si $\operatorname{TrM}_{\mathrm{e}}=0$, y los niveles de esfuerzo y biomasa están en el interior de la curva de extinción, el esfuerzo de pesca $E$ es regulado por la disminución o aumento de la biomasa, oscilando esta última alrededor del valor $\mathrm{x}_{\mathrm{e}} \mathrm{y}$ el esfuerzo en tomo al valor $\mathrm{E}_{\mathrm{e}}$ dado en Clark (1985)

En caso contrario, i.e., si los niveles de esfuerzo y biomasa están fuera de esta curva, la pesquería colapsa, es decir, existe un valor máximo de esfuerzo $\mathrm{E}_{\mathrm{u}}$, para el cual es posible regular la pesquería (Fig. 1d).

\section{Bioteorema 3}

a) $\mathrm{Si} \operatorname{TrM}$ > 0 y el cuociente (a b $\pi / 1)$ estácercano al valor de bifurcación $B^{*}$, existe una curva cerrada aislada (cielo límite). Cuando los niveles de esfuerzo están en el interior de la curva de extinción la pesquería puede autorregularse y para niveles sobre $\mathrm{E}_{\mathrm{u}}$ la pesquería colapsa (Fig. $2 \mathrm{~b}$ ).

b) $\operatorname{Si} \operatorname{TrM}_{\mathrm{e}}>0$ y si b(q $\left.\pi-\mathrm{cl}\right)-1 \gamma<0$, no existe cielo límite y el punto de equilibrio es inestable e implica que la biomasa de peces se extingue para cualquier nivel de explotación (Fig. 2b). 

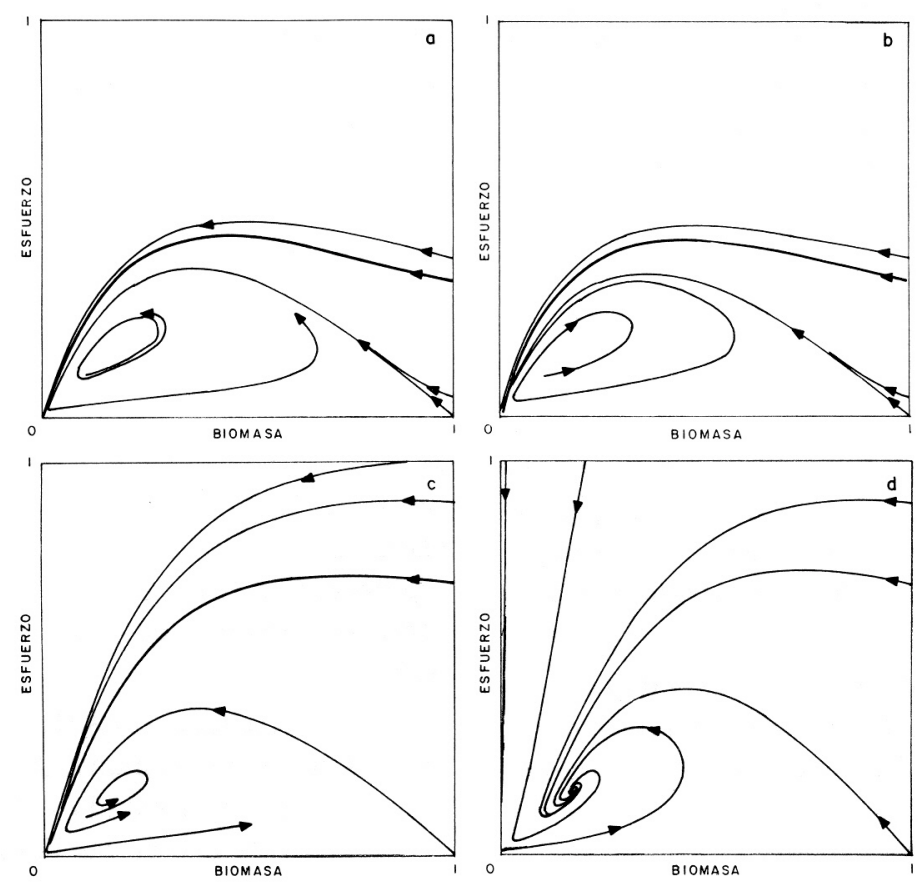

Figura 2. a) Autorregulación oscilatoria y curva de extinción $(a=0,6 ; b=0,58 ; c=0,5) ; b)$ colapso de la pesquería $(a=0,6 ; b=0,5 ; c=0,5) ; c)$ autorregulación y curva de extinción $(a=0,6 ; b=0,7 ; c=0,5) ; y$ d) autorregulación $(a=0,6 ; b=1,2 ; c=0,5)$.

\section{Bioteorema 4}

a) Cuando $\operatorname{Tr} \mathrm{M}_{\mathrm{e}}<0$ y además b(q $\left.\pi-\mathrm{cl}\right)-1 \gamma<0$, para niveles de esfuerzo por debajo de la curva de extinción, se tiene que la pesquería tiende a estabilizarse al punto de equilibrio no trivial, mientras que para condiciones iniciales sobre la curva, la pesquería colapsa (Fig. 2c).

b) $\operatorname{Si~}_{\operatorname{TrM}}<0, \mathrm{~b}(\mathrm{q} \pi-\mathrm{cl})-1 \gamma \quad 0$ y a b $\pi<1$, cualquiera sea el esfuerzo E que se haga, éste tiende a $E_{e}$ y la biomasa tiende a $x_{e}$ (Fig. 2d).

Salvo en los casos 1a y $4 \mathrm{~b}$, en todas las otras situaciones se observa que para un mismo nivel de la biomasa, si los niveles de esfuerzo están por sobre un nivel umbral $E_{u}$ implica que la pesquería colapsa. Para niveles de esfuerzo bajo $\mathrm{E}_{\mathrm{u}}$ se puede mantener autorregulada en el largo plazo cuando el parámetro $B$ está cerca del valor de bifurcación $\mathrm{B}^{*}$ pero si $\mathrm{B}$ es pequeño el equilibrio positivo del modelo es inestable y por lo tanto la población se extingue.

González y Mena (1991a) han analizado la estabilidad y periodicidad del sistema reparametrizado comprobándose la existencia de ciclos o trayectorias cerradas para ciertos valores de los parámetros.

La existencia de tales ciclos son de importancia en el modelo, puesto que ello indica que para ciertas condiciones iniciales y para algunos valores de las constantes involucradas, la pesquería se podría autorregular, pero lamentablemente estos ciclos coexisten con situaciones en que para determinados niveles del esfuerzo, la población de peces va a la extinción.

\section{DISCUSION}

La situación descrita en el primer bioteorema indica que la pesquería colapsa, aun cuando el equilibrio esté fuera del primer cuadrante, lo que indica que las condiciones biológicas y de mercado son inadecuadas.

En la actualidad parece poco realista que una biomasa explotada comercialmente, se mantenga estable en el largo tiempo como lo expresa el caso a) del último bioteorema. 
Es importante destacar la existencia de una curva de extinción en casi todos los casos analizados, por cuanto ella indica los niveles sobre los cuales cualquier esfuerzo de pesca lleva al agotamiento del recurso. Esta situación puede darse incluso si la biomasa está en niveles cercanos a su nivel máximo $\mathrm{K}$.

Al mantener fijo los valores de A y $\mathrm{C}$ se obtuvo como punto de bifurcación el valor $\mathrm{B}^{*}=\frac{1-\mathrm{C}^{2}-\mathrm{A}}{\mathrm{C}(1-\mathrm{C})}$, que en relación a los parámetros originales corresponde a variar $b$, la constante de rigidez, la cual mide la flexibilidad con que los inversionistas modifican su esfuerzo de pesca $\mathrm{E}$ (las embarcaciones que envían a la captura). En las Figuras $1 \mathrm{~d}$ y $2 \mathrm{a}$ a $2 \mathrm{~d}$ se eligió $\mathrm{A}=0.6$ y $\mathrm{C}=0.5$ resultando $\mathrm{B}=0.6$ como punto de bifurcación.

Suponer todas las constantes fijas, salvo b (i.e., las constantes A y $\mathrm{C}$ del sistema desparametrizado son fijas), corresponde a suponer que son invariantes las características propias de la especie explotada (y y K), la política de precio, impuesto y mercado, los costos y las técnicas de captura (p, T, q, a, 1 y c fijos).

El parámetro b mide la disponibilidad de capital y el acceso a la información acerca del estado de la pesquería que en un momento determinado disponen los inversionistas y este parámetro es el que se hace variar en el estudio computacional que se muestra en las figuras adjuntas.

1) Si b es grande significa que los inversionistas pueden percibir en pequeños lapsos de tiempo el comportamiento de su ingreso y reaccionar rápidamente con la información obtenida; esto le permite adoptar nuevas decisiones con respecto a la utilización de su capital, es decir, con respecto al nivel de esfuerzo de pesca que debe realizar para mantener su nivel de ingreso.

2) Si b es pequeño, la reacción con respecto al esfuerzo que debe realizar es más lenta en el tiempo. En esta situación hay niveles de esfuerzo para los cuales la biomasa se extingue. Este problema no es detectado por los inversionistas (industriales), por tener una lenta reacción, y siguen enviando sus embarcaciones a pescar, aun cuando el nivel de esfuerzo sea mayor que el umbral de extinción $\mathrm{E}_{\mathrm{u}}$; en consecuencia sus ingresos son escasos y se reducirán a cero en poco tiempo.

Este comportamiento de la pesquería refleja lo que sucede con la pesca de orilla, efectuada princi- palmente por pescadores artesanales. Si se considera un tamaño fijo de la biomasa existente, se puede observar numéricamente que un esfuerzo de pesca por sobre un cierto nivel umbral la población de peces se extingue.

Esto indica, por ejemplo, que el ingreso de grandes embarcaciones a la pesca costera se debería condicionar a que su esfuerzo de pesca sea menor que el umbral numéricamente obtenido. Las Figuras lb, 1d, 2a y 2c muestran que existen los umbrales de esfuerzo $\mathrm{E}_{\mathrm{u}}$, para un determinado nivel de la biomasa y para el respectivo valor de b. Como la pesca industrial proviene de grandes capitales significa que si los esfuerzos no son controlados por una política de regulación, fácilmente alcanzarían magnitudes por sobre los umbrales indicados en las figuras mencionadas.

Si x representa el stock (fracción explotable de la población) y el rendimiento de pesca es el cuociente entre la biomasa capturada y el esfuerzo de pesca se puede observar que en las Figuras 3 y 6 muestran que para bajos niveles de esfuerzo, se obtiene altos rendimientos, lo cual induce a pensar que la pesquería es buena, pero se ve claramente que esta explotación lleva al colapso de la población de peces.

De las Figuras 1b, 1d, 2a y 2c se concluye que el rendimiento óptimo se obtiene cuando el recurso pesquero es mejor conservado, puesto que en esas condiciones, los niveles de esfuerzo deben mantenerse bajo la curva de extinción.

Se puede concluir en forma general que, un bajo rendimiento de la pesca, lleva al agotamiento de la pesquería, porque el esfuerzo de pesca está por sobre la curva de extinción.

\section{CONCLUSIONES}

En el modelo analizado se asumen ciertos supuestos que no pueden ser aplicados a cualquier tipo de pesquería. Se deduce claramente que aplicar la hipótesis de Schaefer en esas mismas pesquerías, tampoco es un postulado adecuado.

Esto implica que al suponer variable el coeficiente de captura, haciéndolo dependiente tanto de la biomasa como del esfuerzo de pesca, pero asumiendo otra relación entre ellos, se da origen a otro modelo diferente, para el cual el comportamiento cualitativo podría resultar muy distinto. 
Puesto que en la realidad las pesquerías comerciales se comportan de una manera más bien fluctuante, esta situación corresponde a considerar que en el modelo el punto de equilibrio positivo no es estable, situación que es descrita en los bioteoremas 2 y 3 (Figs. 1d, 2a y 2b).

Los parámetros para los cuales se cumple la autorregulación (dando oportunidad a la recuperación de la biomasa) deben ser estimados por los biólogos pesqueros u otros interesados en el tema, pero puede inferirse claramente del análisis matemático realizado, que una pesquería de acceso abierto con la función de captura que se asume, corre fuertes riesgos de ser sobrexplotada cuando se realizan esfuerzos de pesca por sobre los niveles umbrales (curva de extinción) y aunque este esfuerzo sea pequeño.

Esto demuestra algo ya conocido por los estudiosos del tema, por lo que se refuerza la conveniencia de un control sobre la pesquería, por parte de una agencia reguladora. Como se indica en el modelo presentado en (Chaudhuri y Johnson, 1990), este control puede ser a través de los impuestos a la cantidad de biomasa desembarcada, y de esta manera llevar los niveles del esfuerzo de pesca bajo los valores dados por la curva de extinción. Esta regulación, en opinión de los economistas resulta ser más efectiva, al menos en teoría, a otras políticas de control debido a su flexibilidad y muchas de las ventajas de un sistema económico de competencia pueden ser mejor mantenidas.

Resulta de suma importancia la información acerca de todas las variables que están involucradas en el proceso pesquero, como son, por ejemplo, la dinámica de la población (el conocimiento de su período de desove, la zona en que éste se produce, etc.) y la seguridad económica de la inversión (costo de oportunidades). Todo esto hace que el parámetro b sea más grande y por lo tanto la pesquería se hace más estable permitiendo la protección del recurso explotado, lo que debe ser un objetivo compartido por todos los interesados en esta actividad.

En resumen, el modelo analizado da cuenta de diversas situaciones que suceden frecuentemente en pesquerías, aunque se asuman varias simplificaciones respecto a la población de peces y al esfuerzo de pesca. Su validación dependerá del aporte que entreguen los biólogos pesqueros o los economistas, dado que su aplicación a la modelación biopesquera es notoria.

\section{AGRADECIMIENTOS}

Los autores desean manifestar sus agradecimientos a los profesores Jorge González G. y Raúl Naulín L., por las sugerencias formuladas al presente trabajo, durante las exposiciones realizadas en el Taller de Ecología Matemática que funciona en el Instituto de Matemáticas de la Universidad Católica de Valparaíso y al Sr. René Cerda D., profesor de la Escuela de Ciencias del Mar de la misma Universidad, por sus valiosas observaciones.

\section{REFERENCIAS BIBLIOGRAFICAS}

ARROWSMITH, D.K. and C.M. PLACE. 1982. Ordinary differential equations. Chapman and Hall, 154 pp.

CLARK, C.W. 1990. Mathematical Bioeconomic: The optimal management of renewable resources. John Wiley and Sons, 386 pp.

CLARK, C.W. 1979. Mathematical models in the economics of renewable resources. SIAM Review 21 (1): 81-99.

CLARK, C.W. 1985. Bioeconomic modelling and fisheries management. John Wiley and Sons, 291 pp.

CHAUDHURI, K. and T. JOHNSON. 1990. Bioeconomic dynamics of a fishery modeled as an S-System. Math. Biosc., 99: 231-249.

DUMORTIER, F. 1978. Singularities of vector fields. Monografías de Matemáticas No. 32, Instituto de Matemática Pura e Aplicada, Brasil, 191 pp.

GONZALEZ, E. 1991. El modelo de Smith para una pesquería de acceso abierto. Una revisión crítica, Anuario Instituto de Matemáticas, Universidad Católica de Valparaíso, pp. 83-92.

GONZALEZ, E. y J. MENA. 1991a. Dinámica de un modelo bioeconómico de una sola especie. Actas del 63 Encuentro de la Sociedad Matemática de Chile, pp. 85-89.

GONZALEZ, E. y J. MENA. 1991b. Periodicidad y estabilidad en un modelo de pesquería de una sola especie. Actas del V Congreso Internacional de Biomatemáticas, $50 \mathrm{pp}$. 
GONZALEZ, E. y J. MENA. 1992. Existencia de ciclos límites en un modelo de pesquería. Actas del Segundo Encuentro de Matemáticas Capricornio, pp. 17-19.

HASSARD, RD; ND. KAZARINOFF and Y-H. WAN. 1981. Theory and applications of Hopf bifurcation. Lecture Notes Series 41, London Mathematical Society, Cambridge University Press, $311 \mathrm{pp}$.

JORDAN, D.W. and P. SMITH. 1983. Nonlinear ordinary differential equations. Clarendon Press Oxford, $360 \mathrm{pp}$.

KOÇAK, H. 1986. Differentia1 and difference equations through computer experiments. Springer- Verlag, 225 pp.

MENA, J. 1988. Periodicity and stability in epidemiological models with disease-related deaths. Ph.D. Thesis, University of Iowa, 98 pp.

SAEZ E. and I. SZANTO. 1990. An existence of inequality of a fine focus. IEEE Transactions on Circuity and Systems, 37 (5): 661-663.

YAÑEZ, E. y L. MARITANO. 1983. Análisis histórico (1971-1980) de la pesquería pelágica de la zona norte de Chile $\left(18^{\circ} 20^{\prime} \mathrm{S}-24^{\circ} 00^{\prime} \mathrm{S}\right)$ y estimación del estado de los recursos explotados. En: P. Arana (ed.). Análisis de Pesquerías Chilenas. Escuela de Ciencias del Mar, UCV, Valparaíso, pp. 161-177.

\section{ANEXO 1}

En el sistema de ecuaciones diferenciales se analiza matemáticamente en los puntos de equilibrio del primer cuadrante $G=\left(R^{+}\right)^{2}$. En el estudio se utiliza el análisis local de los puntos hiperbólicos (Criterio de RouthHurwitz), el Teorema de Poincaré-Bendixson, el Test de Dulac, bifurcaciones de Hopf, la transformada de Poincaré y el método del Blowing-up.

$$
\begin{aligned}
& \dot{x}=\left[A(1-x)-\frac{E}{E+x}\right] x \\
& \dot{E}=B\left[-C+\frac{E}{E+x}\right] E
\end{aligned}
$$

Los puntos de equilibrio ocurren cuando la biomasa y el esfuerzo son ambos nulos, también cuando la biomasa no es explotada y está en su nivel máximo y además en el punto de equilibrio no trivial (equilibrio bionómico Clark, 1990), cuando los niveles de biomasa y esfuerzo son

$$
x_{e}=\frac{A-(1-C)}{A} \text { y } E_{e}=\frac{1-C}{C} x_{e}
$$

Para que el equilibrio positivo esté en primer cuadrante, se debe cumplir simultáneamente que

$$
A-(1-C)>0 \text { y } 1-C>0
$$

En el espacio de los parámetros se define el conjunto:

$$
Q_{e}=\left\{(A, B, C) \in R^{3} / C<1 \Lambda A>1-C>0\right\}
$$

que satisface tales condiciones. Al tomar valores de los parámetros en $\mathrm{Q}_{\mathrm{e}}$ se tiene que la región

$$
\mathrm{K}_{\mathrm{e}}=\left\{(\mathrm{x}, \mathrm{E}) \in \Gamma / \mathrm{x} \leq 1 \Lambda \mathrm{E} \leq \frac{1-\mathrm{c}}{\mathrm{c}}\right\}
$$

es positivamente invariante en el espacio de fase (González y Mena, 1991b), es decir, toda trayectoria del sistema que comience en $\mathrm{K}_{\mathrm{e}}$, permanece en él, o sea, la relación entre la biomasa y el esfuerzo siempre están en el conjunto.

$\mathrm{Si} \mathrm{M}_{\mathrm{e}}$ indica la matriz jacobiana del campo (3), (matriz de las derivadas parciales) en $\mathrm{P}_{\mathrm{e}}=$ $\left(\mathrm{x}_{\mathrm{e}}, \mathrm{E}_{\mathrm{e}}\right)$ se obtiene que su polinomio característico es

$$
\begin{array}{ll}
\operatorname{det}\left(M_{e}-p I_{2}\right)=P(p)=p^{2}-\operatorname{TrM}_{e} p+\operatorname{det} M_{e} \\
\text { donde } & \operatorname{TrM}_{e}=1-C^{2}+B C^{2}-B C-A \\
& \operatorname{det} M_{e}=A B C^{2} E_{e}>0
\end{array}
$$

por lo tanto, sólo el signo de la traza determina el signo de la parte real de las raíces de este polinomio (valores propios de la matriz). González y Mena, (1991a) prueban que:

1) $\mathrm{Si} \operatorname{TrM}_{e}<0$, el punto $P_{e}$ es localmente estable y existe curva de extinción (Fig. 2c). Si se cumple que $\mathrm{B}(1-\mathrm{C})>\mathrm{A}$ y $\mathrm{B} \leq 1$, el punto $\mathrm{P}$ es global asintóticamente estable en $\mathrm{K}$ y la curva de extinción no existe (Fig. 2d).

2) Si $\operatorname{TrM}_{e}>0$, se cumple que A y B $<1$ y el punto $\mathrm{P}_{\mathrm{e}}$ es inestable; existe al menos una órbita periódica en tomo a $\mathrm{P}_{\mathrm{e}}$ que coexiste con una cur- 
cuando el valor de $\mathrm{B}$ es cercano al valor de bifurcación $\mathrm{B}^{*}$ (Fig. la).

Si B $(1-C)<$ A (B es pequefio) el punto $(0,0)$ resulta ser un atractor global (Fig. lb).

3) $\mathrm{Si} \operatorname{TrM}=0$ se demuestra también la existencia de órbitas periódicas en torno a $\mathrm{P}_{\mathrm{e}} \mathrm{y}$ de una curva de extinción (Fig. 1d). Se obtiene $B^{*}=\frac{1-C^{2}-A}{C(1-C)}$ como punto de bifurcación.

En este último caso se tiene que sobre la superficie

$$
A=(1-C)(1+C-B C)
$$

del espacio de los parámetros, el punto de equilibrio situado en el primer cuadrante es un centro foco débil (Sáez y Szantó, 1990). Mediante el cálculo de las derivadas de la transformación de Poincaré, se puede contar los. ciclos límites cuya existencia está garantizada por el teorema de Poincaré y las bifurcaciones de Hopf.

Puesto que el punto $(0,0)$ es de carácter no hiperbólico, su análisis se efectúa con las técnicas del blowing-up (Dumortier, 1978), determinando la dirección de entrada de la curva de extinción. Esta curva se define como el lugar geométrico de los puntos en que la dirección del vector del campo en el punto $(0,0)$ está dada por el ángulo

$$
\alpha=\arctan \left(\frac{\mathrm{A}-\mathrm{B}(1-\mathrm{C})}{1-\mathrm{A}-\mathrm{BC}}\right)
$$

Recibido el 3 de marzo de 1993. Aceptado el 5 de octubre de 1994.
Este punto tiene características especiales, por cuanto si el punto de equilibrio positivo no es estable, o bien, si es localmente estable, esto es, A > B (1-C), bajo ciertas condiciones iniciales se comporta como atractor, lo cual indica que para niveles de esfuerzo que superan un valor umbral determinado por el ángulo $\alpha$, la biomasa se extingue.

Para $\mathrm{P}_{\mathrm{e}}$ no está en el primer cuadrante se debe cumplir una de las siguientes relaciones:

$$
\begin{aligned}
& \text { i) } \mathrm{A}-(1-\mathrm{C})<0 \text {, ó } \\
& \text { ii) } 1-\mathrm{C}<0
\end{aligned}
$$

Luego, $\mathrm{P}_{\mathrm{e}}$ puede estar sólo en el tercer o cuarto cuadrante. En la primera situación, el punto $(1,0)$ resulta ser un repulsor, puesto que el polinomio característico $\mathrm{P}(\mathrm{p})$ asociado a su matriz jacobiana, tiene un valor propio con parte real positiva y otro con parte real negativa. El punto $(0,0)$ es un atractor global y todas las trayectorias que comienzan en la región $\mathrm{K}_{\mathrm{e}}$, terminan en este punto (Fig. lc).

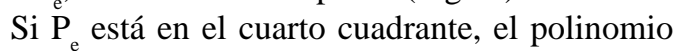
$\mathrm{P}(\mathrm{p})$ asociado a su matriz jacobiana, tiene los dos valores propios con parte real negativa, lo cual indica que $(1,0)$ es atractor local. Se tiene dos situaciones distintas para este caso:

Si $B \geq 1$, el punto $(1,0)$ resulta ser atractor global (Fig. la), mientras que si $\mathrm{B}<1$, existe una curva de extinción y el punto $(0,0)$ es también atractor para las trayectorias que pasan por puntos ubicados sobre esa curva (Fig. 1b). 